\title{
RADIO FREQUENCY INTERFERENCE DETECTION FOR PASSIVE REMOTE SENSING USING EIGENVALUE ANALYSIS
}

\author{
Adam J. Schoenwald ${ }^{1,2}$, Seung-Jun Kim ${ }^{1}$, Priscilla N. Mohammed ${ }^{2,3}$ \\ ${ }^{1}$ University of Maryland Baltimore County, Baltimore, MD \\ ${ }^{2}$ NASA Goddard Space Flight Center, Greenbelt, MD \\ ${ }^{3}$ Goddard Earth Sciences Technology and Research, Morgan State University
}

\begin{abstract}
Radio frequency interference (RFI) can corrupt passive remote sensing measurements taken with microwave radiometers. With the increasingly utilized spectrum and the push for larger bandwidth radiometers, the likelihood of RFI contamination has grown significantly. In this work, an eigenvaluebased algorithm is developed to detect the presence of RFI and provide estimates of RFI-free radiation levels. Simulated tests show that the proposed detector outperforms conventional kurtosis-based RFI detectors in the low-to-medium interferece-to-noise-power-ratio (INR) regime under continuous wave $(\mathrm{CW})$ and quadrature phase shift keying (QPSK) RFIs.
\end{abstract}

Index Terms - Passive remote sensing, radio frequency interference mitigation, eigenvalue methods.

\section{INTRODUCTION}

Passive remote sensing using microwave radiometry provides a valuable insight into the geophysical state of our environment. Deploying remote sensing instruments on space flight satellites offers an unparalleled capability to observe globalscale system dynamics evolving across the planet. The nature of radiometry calls for sensitive instruments to map the second order statistics (SOS) of the geophysical thermal radiation process to a physical quantity of some scientific value.

Any signal not originating from the physical processes of interest, such as the radio frequency interference (RFI), can offset the SOS measurements, resulting in degraded quality for use in a scientific study. When the interference source is powerful enough, the radiometer measurements may be offset beyond a physically reasonable range, which can be easily tagged and removed from the dataset. A more detrimental scenario is when the RFI is weak enough that the measurement appears plausible yet corrupt, potentially misleading scientific interpretation.

This work was supported in part by NASA Earth Science Technology Office NNH13ZDA001N ACT Funding, and National Science Foundation Grant 1547347.
Obtaining passive radiometry readings that are free of RFI is a non-trivial task. The optimal set of frequency bands for remote sensing changes depending on the nature of the physical parameters being measured. While regulating authorities such as the FCC and the ITU have allocated various frequency bands for passive remote sensing, some bands such as the allocation at $18.7 \mathrm{GHz}$ are shared allocations, where remote sensing and communication applications must coexist. To compound the issue, observing a large swath of bandwidth is often desired to increase the measurement sensitivity.

Existing radiometer missions have experienced RFI issues that can potentially render the collected data unusable. The Soil Moisture and Ocean Salinity (SMOS) mission [1, 2] has experienced RFI at the $1,400 \mathrm{MHz}$ band, and found that, without a RFI mitigation strategy in place, the mission must actively seek out and turn off the sources of interference [3]. The Aqua Satellite's Advanced Microwave Scanning Radiometer (AMSR-E) program has experienced RFI in the $6.9 \mathrm{GHz}$ and $10.7 \mathrm{GHz}$ bands [4].

RFI mitigation algorithms have been developed and used in existing satellite remote sensing missions such as Soil Moisture Active Passive (SMAP) [5, 6]. While existing methods have been useful at flagging narrowband pulsed interference, research has been done to develop more sensitive detectors such as the complex signal kurtosis (CSK) detectors to efficiently flag wideband and continuous interference $[7,8]$.

In this work, inspired by a related approach in a cognitive radio setting [9], the property of the man-made interference to occupy a low-dimensional subspace of the ambient measurement dimension is exploited. The ratio of the maximum to the minimum eigenvalues of the measurement covariance matrix is employed as a test statistic. Also, the RFI-free noise power estimate is obtained from the minimum eigenvalue.

The rest of the paper is organized as follows. The signal model is described in Sec. 2. The eigenvalue-based RFI detection and excision algorithm is developed in Sec. 3. The results from numerical tests are provided in Sec. 4 , and the conclusions in Sec. 5. 


\section{SIGNAL MODEL}

The RFI detection problem is posed as a binary hypothesis testing problem.

$$
\begin{aligned}
& H_{0}: x(t)=w(t) \\
& H_{1}: x(t)=w(t)+r(t)
\end{aligned}
$$

The null hypothesis, $H_{0}$ in (1), represents the case where the received signal $x(t)$ contains only the thermal noise $w(t)$ radiated by the earth. The alternate hypothesis, $H_{1}$ in (2), represents the case where additional man-made interference $r(t)$ is observed by the radiometer. The thermal noise process $w(t)$ is assumed to be a zero-mean Gaussian noise process with variance $\sigma_{w}^{2}$.

The man-made interference $r(t)$, with variance $\sigma_{r}^{2}$, can be modeled using different modulations and channelizations. In this work, three interference types are considered: The continuous wave $(\mathrm{CW})$ interference; the single-channel quadrature phase shift keying (QPSK)-modulated signal pulse-shaped using a root raised cosine (RRCOS) filter; and the combination of multiple channels of QPSK RRCOS signals. In all cases, the knowledge of the carrier frequency is unavailable, resulting in passband signals. No phase synchronization or carrier recovery is attempted.

A single-polarization radiometer using a digital back end is considered, which samples $x(t)$ as $x[k] \equiv x\left(k T_{s}\right)$, where $T_{s}$ is the Nyquist sampling interval, which is related to the bandwidth $B$ of the RFI as $T_{s} \leq 1 / B$. The resulting signal $x[k]$ under $H_{0}$ contains only Gaussian noise samples $w[k]$, while under $H_{1}$ both $w[k]$ and the sampled RFI $r[k]$. That is,

$$
\begin{aligned}
& H_{0}: x[k]=w[k] \\
& H_{1}: x[k]=w[k]+r[k] .
\end{aligned}
$$

\section{EIGENVALUE-BASED RFI ALGORITHMS}

\subsection{Eigenvalue-Based RFI Detector}

The maximum-to-minimum eigenvalue (MME) ratio test proposed in the cognitive radio context in [9] is adapted here to passive microwave remote sensing. The key idea is that manmade interference signals typically occupy low-dimensional subspace after oversampling.

Assume that the signal is oversampled by a factor of $M$, that is, $T_{s}=1 /(B M)$. Then upon defining

$$
x_{i}(n) \equiv x[n M+i-1], \quad i=1,2, \ldots, M
$$

the $n$-th block of samples are put together into a vector $\mathbf{x}(n)$ as

$$
\mathbf{x}(n) \equiv\left[x_{1}(n), x_{1}(n), \ldots, x_{M}(n)\right]^{T}
$$

where ${ }^{T}$ denotes transposition. Collecting $L$ blocks, one obtains

$$
\hat{\mathbf{x}}(n) \equiv\left[\mathbf{x}^{T}(n), \mathbf{x}^{T}(n-1), \ldots \mathbf{x}^{T}(n-L+1)\right]^{T} \in \mathbb{C}^{L M}
$$

where $L$ is a smoothing factor that can be used to increase the dimensionality for subspace extraction.

The sample covariance matrix $\mathbf{R}_{x}\left(N_{s}\right)$ is obtained as

$$
\mathbf{R}_{x}\left(N_{s}\right) \equiv \frac{1}{N_{s}} \sum_{n=L-1}^{L-2+N_{s}} \hat{\mathbf{x}}(n) \hat{\mathbf{x}}(n)^{\dagger}
$$

where $N_{s}$ is the number of sample vectors and $\dagger$ denotes Hermitian transpose. The eigenvalues of $\mathbf{R}_{x}\left(N_{s}\right)$ are then calculated and used to form a test statistic. The ratio between the maximum and the minimum eigenvalues forms the MME ratio test statistic $\mathbf{T}_{\lambda}$ given by

$$
\mathbf{T}_{\lambda}=\frac{\lambda_{\max }}{\lambda_{\min }} .
$$

Numerical simulations of this proposed method are shown in Sec. 4.1.

\subsection{Eigenvalue-Based RFI Excision}

In addition to detecting the RFI, the power of the Gaussian noise can be estimated based on the assumption that the dimension of the RFI subspace is smaller than the ambient dimension. A simple scheme is to use $\lambda_{\min }$, the minimum eigenvalue of $\mathbf{R}_{x}\left(N_{s}\right)$ from (8) to estimate the noise power $\sigma_{w}^{2}$. Under the null hypothesis $H_{0}$, the estimate $\hat{\sigma}_{w}^{2}$ for the noise power is given by

$$
\hat{\sigma}_{w}^{2}=\lambda_{\min } \frac{N_{s}}{\left(\sqrt{N_{s}}-\sqrt{M L}\right)} .
$$

The scaling factor in (10) is justified from the limiting distribution of the eigenvalues. With $y \equiv \lim _{N_{s} \rightarrow \infty} \frac{M L}{N_{s}} \in(0,1)$, the extreme eigenvalues of the sample covariance matrix converge almost surely to [10]

$$
\begin{gathered}
\lim _{N_{s} \rightarrow \infty} \lambda_{\min }=\sigma^{2}(1-\sqrt{y})^{2} \\
\lim _{N_{s} \rightarrow \infty} \lambda_{\max }=\sigma^{2}(1+\sqrt{y})^{2} .
\end{gathered}
$$

Provided that $N_{s}$ is relatively large, the observed $\lambda_{\min }$ can be used to find $\hat{\sigma}_{w}^{2}$ using the scaling factor derived from (11) and shown in (10). This concept is extended to the estimation of $\sigma_{w}^{2}$ under the alternate hypothesis $H_{1}$ when RFI is present. If RFI occupies a low-dimensional subspace, the energy of the Gaussian noise process will be captured by the lower set of eigenvalues. Numerical tests of this method in Sec. 4.2 verify acceptable performance of this approach.

\section{NUMERICAL TESTS}

\subsection{RFI Detection}

To evaluate the performance of the proposed detector under various conditions, a receiver operating characteristic (ROC) 




Fig. 1. AUC performance of the proposed detector.

curve was obtained through Monte-Carlo simulations, and the area-under-curve (AUC) metric was used as the figure of merit. The interference-to-noise power ratio (INR) in decibels is defined as

$$
\mathrm{INR}_{\mathrm{dB}}=10 \log _{10} \frac{\sigma_{r}^{2}}{\sigma_{w}^{2}} .
$$

The plot of the AUC values at different INR levels is shown in Fig. 1, which was obtained from 200 Monte-Carlo runs. The total number of samples was $N=10,000$, with parameters $M=4$ and $L=4$. The performance of the MME ratio-based detector was compared to those of the full-band kurtosis-based detector [5], as well as the spectral kurtosis detector based on 16 sub-bands [11]. It can be seen from Fig. 1 that the MME ratio test outperforms the detectors based on kurtosis and spectral kurtosis.

\subsection{Noise Power Estimation}

To evaluate the noise power estimation performance, the mean square error (MSE) was calculated between the proposed estimate and the true noise power used to generate the dataset, which was $\sigma_{w}^{2}=1$. The MSE from the sample variance was calculated for comparison.

Fig. 2 shows the MSE values as the INR is varied for different RFI models considered. It can be seen from the figure that the proposed eigenvalue-based noise power estimator outperforms the sample variance estimator in the medium-tohigh INR regime, while at low SNR levels, the sample variance yields smaller MSE. Therefore, a hybrid approach will be explored in the future work. Furthermore, the MSE performance of the eigenvalue-based noise power estimates vary for different signal types. This may be because the dimension of the subspace occupied by the RFI changes depending on the RFI types. Future work will examine the use of an order selection method to determine the dimension of the noise

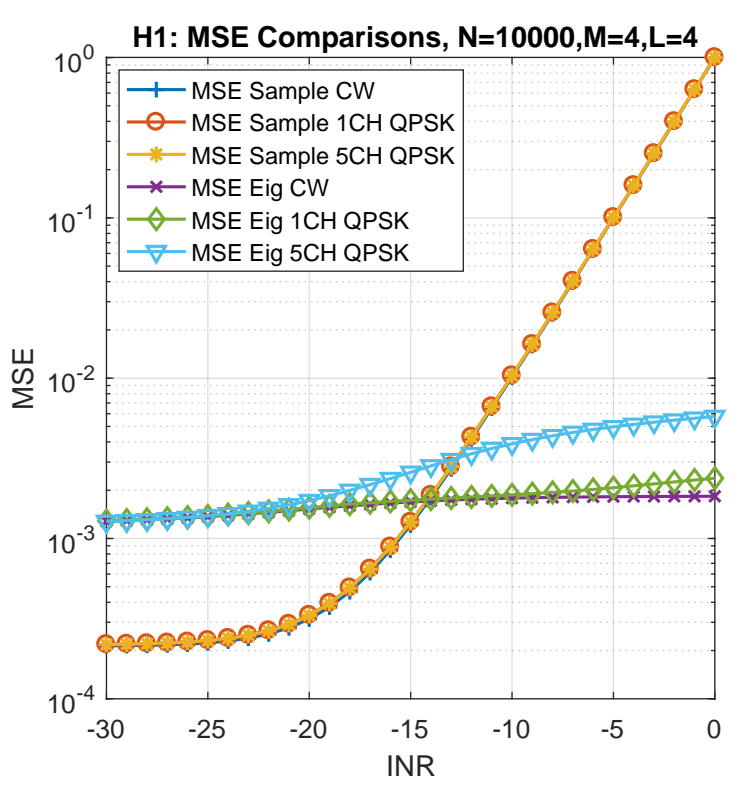

Fig. 2. MSE of the noise power estimates.

subspace, and averaging multiple eigenvalues to improve the estimation performance.

\section{CONCLUSION}

An RFI detector based on the ratio of the maximum to the minimum eigenvalues of the signal covariance matrix has been proposed for passive remote sensing. The power level of the RFI-free thermal radiation was estimated as the scaled minimum eigenvalue. Numerical tests verified that the proposed detector yields superior detection capability over the full-band and spectral kurtosis-based RFI detectors under CW, QPSK, and multi-channel QPSK RFIs, especially in the low-to-medium INR ranges. The eigenvalue-based noise power estimator outperformed the sample covariance estimate in the medium-to-high INR ranges.

In future work, we will focus on the eigenvalue methods for both detection and estimation. Further analysis/experiments will be done to compare the MME detector with other stateof-the-art detection methods. The ability to improve the accuracy of the noise power estimation will be explored. Development of a joint detection/estimation algorithm and the use of an order-selection method seem to be promising directions.

\section{REFERENCES}

[1] J. Font, A. Camps, A. Borges, M. Martín-Neira, J. Boutin, N. Reul, Y. H. Kerr, A. Hahne, and S. Mecklenburg, "SMOS: The challenging sea surface salinity 
measurement from space," Proc. IEEE, vol. 98, no. 5, pp. 649-665, 2010.

[2] Y. H. Kerr, P. Waldteufel, J.-P. Wigneron, S. Delwart, F. Cabot, J. Boutin, M.-J. Escorihuela, J. Font, N. Reul, C. Gruhier, et al., "The SMOS mission: New tool for monitoring key elements of the global water cycle," Proc. IEEE, vol. 98, no. 5, pp. 666-687, 2010.

[3] R. Oliva, E. Daganzo, Y. H. Kerr, S. Mecklenburg, S. Nieto, P. Richaume, and C. Gruhier, "SMOS radio frequency interference scenario: Status and actions taken to improve the rfi environment in the $1400-1427-\mathrm{MHz}$ passive band," IEEE Trans. Geosci. Remote Sens., vol. 50, no. 5, pp. 1427-1439, May 2012.

[4] E. G. Njoku, P. Ashcroft, T. K. Chan, and L. Li, "Global survey and statistics of radio-frequency interference in AMSR-E land observations," IEEE Trans. Geosci. Remote Sens., vol. 43, no. 5, pp. 938-947, May 2005.

[5] R. D. De Roo, S. Misra, and C. S. Ruf, "Sensitivity of the kurtosis statistic as a detector of pulsed sinusoidal RFI," IEEE Trans. Geosci. Remote Sens., vol. 45, no. 7, pp. 1938-1946, Jul. 2007.

[6] J. R. Piepmeier, J. T. Johnson, P. N. Mohammed, D. Bradley, C. Ruf, M. Aksoy, R. Garcia, D. Hudson, L. Miles, and M. Wong, "Radio-frequency interference mitigation for the soil moisture active passive microwave radiometer," IEEE Trans. Geosci. Remote Sens., vol. 52, no. 1, pp. 761-775, Jan. 2014.

[7] D. Bradley, J. M. Morris, T. Adali, J. T. Johnson, and M. Aksoy, "On the detection of RFI using the complex signal kurtosis in microwave radiometry," in Proc. 13th Specialist Meeting Microwave Radiometry and Remote Sensing of the Environment (MicroRad), Mar. 2014, pp. 33-38.

[8] A. J. Schoenwald, D. C. Bradley, P. N. Mohammed, J. R. Piepmeier, and M. Wong, "Performance analysis of a hardware implemented complex signal kurtosis radiofrequency interference detector," in Proc. 14th Specialist Meeting on Microwave Radiometry and Remote Sensing of the Environment (MicroRad), Apr. 2016, pp. $71-75$.

[9] Y. Zeng and Y. C. Liang, "Eigenvalue-based spectrum sensing algorithms for cognitive radio," IEEE Trans. Commun., vol. 57, no. 6, pp. 1784-1793, Jun. 2009.

[10] Z. D. Bai, "Methodologies in spectral analysis of large dimensional random matrices, a review," Statistica Sinica, vol. 9, no. 3, pp. 611-662, 1999.

[11] G. M. Nita, D. E. Gary, Z. Liu, G. J. Hurford, and S. M. White, "Radio frequency interference excision using spectral-domain statistics," Publications of the Astronomical Society of the Pacific, vol. 119, pp. 805-827, Jul. 2007. 